Musikgeschichte der Hohenlohischen Residenzstadt Kirchberg 
QUELLEN UND STUDIEN ZUR MUSIK IN BADEN-WÜRTTEMBERG

herausgegeben von der

GESELLSCHAFT FÜR MUSIKGESCHICHTE

IN BADEN-WÜRTTEMBERG e.V.

Band 5 


\section{Ingeborg Wesser}

\section{Musikgeschichte \\ der Hohenlohischen \\ Residenzstadt \\ Kirchberg \\ Von der Mitte des 17. bis \\ zum Beginn des 19. Jahrhunderts}

Verlag J. B. Metzler

Stuttgart - Weimar 


\section{GESELLSCHAFT FÜR MUSIKGESCHICHTE IN BADEN-WÜRTTEMBERG e.V.}

Schulberg 2, 72070 Tübingen

Präsident: Hermann Fünfgeld

Vizepräsident: Prof. Dr. Manfred Hermann Schmid

Vorsitzender des Wissenschaftlichen Beirats: Prof. Dr. Manfred Hermann Schmid

Die Deutsche Bibliothek - CIP-Einheitsaufnahme

\section{Wesser, Ingeborg:}

Musikgeschichte der Hohenlohischen Residenzstadt Kirchberg :

Von der Mitte des 17. bis zum Beginn des 19. Jahrhunderts /

Ingeborg Wesser. - Stuttgart ; Weimar, 2001

(Quellen und Studien zur Musik in Baden-Württemberg ; Bd. 5)

ISBN 978-3-476-01845-8

ISBN 978-3-476-02798-6 (eBook)

DOI 10.1007/978-3-476-02798-6

Dieses Werk einschließlich aller seiner Teile ist urheberrechtlich geschützt. Jede

Verwertung außerhalb der engen Grenzen des Urheberrechtsgesetzes ist ohne

Zustimmung des Verlages unzulässig und strafbar. Das gilt insbesondere für Vervielfältigungen, Übersetzungen, Mikroverfilmungen und die Einspeicherung und Verarbeitung in elektronischen Systemen.

(C) 2001 Springer-Verlag GmbH Deutschland

Ursprünglich erschienen bei J. B. Metzlersche Verlagsbuchhandlung

und Carl Ernst Poeschel Verlag GmbH in Stuttgart 2001

www.metzlerverlag.de

info@metzlerverlag.de 


\section{Vorwort}

Ausgangspunkt der vorliegenden Veröffentlichung, die im Sommer 1999 vom Fachbereich Germanistik und Kunstwissenschaften der PhilippsUniversität Marburg als Dissertation angenommen wurde, war die Beschäftigung mit dem Leben und Werk Carl Ludwig Junkers, des hohenlohischen Pfarrers, der sich in zahlreichen Schriften zur Musik seiner Zeit sowie zu ästhetischen Themen, auch der bildenden Kunst, äußerte, Klavier und Flöte spielte und komponierte. Bei der Erkundung von Junkers biographischem Umfeld bin ich im Hohenlohe-Zentralarchiv Neuenstein auf das reiche Quellenmaterial zur Musikgeschichte Kirchbergs gestoßen, das mir einer Aufarbeitung wert schien. Ich möchte meinem akademischen Lehrer, Herrn Professor Dr. Ulrich Siegele, meinen herzlichen Dank aussprechen für die Ermutigung, den hilfreichen Beistand und fachkundigen Rat, mit dem er die Fortschritte des Projekts Kirchberg begleitet hat.

Mein Dank gilt auch Frau Professor Dr. Christel Köhle-Hezinger, Jena, damals Marburg, die mich auf das Graduiertenkolleg »Kunst im Kontext« an der Philipps-Universität Marburg aufmerksam gemacht und den ersten Kontakt hergestellt hat.

Besonders herzlich danke ich Frau Professor Dr. Sabine Henze-Döhring, der Betreuerin meiner Arbeit am Musikwissenschaftlichen Institut der Philipps-Universität Marburg, für ihre wertvollen Ratschläge und Anregungen sowie das Interesse, mit dem sie dieses Stück südwestdeutscher lokaler Musikgeschichte aufgenommen hat.

Danken möchte ich auch den Mitarbeiterinnen und Mitarbeitern der Württembergischen Landesbibliothek Stuttgart, des Landeskirchlichen Archivs Stuttgart, des Hauptstaatsarchivs Stuttgart, des Staatsarchivs Ludwigsburg und vor allem des Hohenlohe-Zentralarchivs Neuenstein. Ein besonderes Anliegen ist es mir, Herrn Wilfried Beutter, HohenloheZentralarchiv Neuenstein, zu danken, der mit seiner Sachkenntnis und seinem landesgeschichtlichen Wissen meine Recherchen wesentlich erleichtert und die Arbeit durch manchen unverhofften Fund bereichert hat. 
Mein Dank geht auch an alle, die mir durch das Lesen des Manuskripts wertvolle Hinweise gaben, und an meinen Sohn Ulrich für seine Hilfe bei der Erstellung der Druckvorlage.

Der »Gesellschaft für Musikgeschichte in Baden-Württemberg e.V.« danke ich für die Aufnahme meiner Dissertation in ihre Veröffentlichungsreihe »Quellen und Studien zur Musik in Baden-Württemberg«.

Stuttgart, im Januar 2001

Ingeborg Wesser 


\section{INHALT}

Vorwort $\ldots \ldots \ldots \ldots \ldots \ldots \ldots \ldots \ldots \ldots \ldots, \mathrm{V}$

Abkürzungen $\ldots \ldots \ldots \ldots \ldots \ldots \ldots \ldots \ldots \ldots \ldots \ldots \ldots \ldots \ldots$

Zur Quellenwiedergabe $\ldots \ldots \ldots \ldots \ldots \ldots \ldots \ldots \ldots \ldots$ XIII

I. Einleitung $\ldots \ldots \ldots \ldots \ldots \ldots \ldots \ldots \ldots \ldots \ldots \ldots$

II. Das Fürstentum Hohenlohe-Kirchberg $\ldots \ldots \ldots \ldots \ldots 5$

A. Das Gesamthaus Hohenlohe und die Linie Kirchberg . . 5

1. Hofstaat und Rangordnung ................ 10

2. Besoldungen ........................ 19

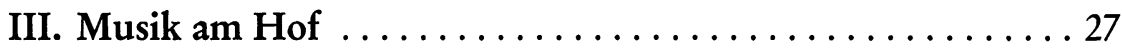

A. Die Hofmusik während der Regierungszeit von Graf Joachim Albrecht 1650-1675 . . . . . . . . . . 27

1. Joachim Albrecht .................... 27

2. Kammermusik und Tafelmusik unter Christian Kneller und Johann Samuel Welter ................. 30

B. Die Hofmusik während der Regierungszeit von Graf Friedrich Eberhard 1701-1737 und Graf, seit 1764 Fürst Carl August 1737-1767 . . . . . . . . . . . 39

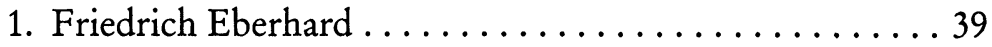

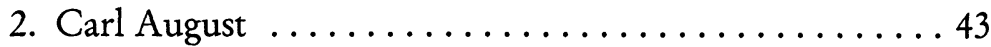

3. Der Hof- und Stadtmusikant Johann Michael Beuerlein .......................47

4. Hofmusik und Hofdivertissements ............ 53

C. Die Hofmusik während der Regierungszeit von Fürst Christian Friedrich Carl 1767-1815 ............ 59

1. Christian Friedrich Carl ................. 59

2. Stadtmusikant und Hoftrompeter, zwei aussterbende Berufe ............................. 65 
3. Aufbau einer Hofkapelle in den Jahren $1768-1775$. . . . 74

a) Instrumente und Notenmaterial . . . . . . . . 75

b) Die Musiker.................... 78

4. Aufschwung der Hofmusik unter Johann Martial

Greiner von 1775-1784 . . . . . . . . . . . . . . . . . . 99

a) Die Aufgaben des Musikdirektors ............ 101

b) Johann Martial Greiner, Geiger und Musikdirektor 106

c) Die Mitglieder der Hofkapelle . . . . . . . . . . 117

d) Hoffeste . . . . . . . . . . . . . . . . . . . 123

e) Öffentliche Hofkonzerte . . . . . . . . . . . . . . 127

f) Das Repertoire der Hofkapelle ........... 131

g) Ein besonderer Fall: Carl Ludwig Junker, Pfarrer,

Schriftsteller, Komponist, Klavierspieler und

Flötist, Chronist des Kirchberger Musiklebens . . . 139

5. Hofmusik unter Präzeptor Johann Georg Beuerlein

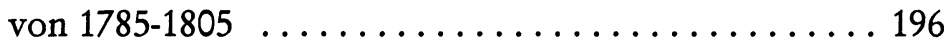

a) Hofdivertissements . . . . . . . . . . . . . . . 197

b) Das Repertoire der Hofkapelle . . . . . . . . . . . 200

c) Das Ende der Hofmusik ............... 206

6. Reisende Musikanten und Virtuosen ............ 211

IV. Musik für die Bürger $\ldots \ldots \ldots \ldots \ldots \ldots \ldots \ldots \ldots \ldots \ldots$

A. Die Dienste des Türmers, Hof- und Stadtmusikanten für die Stadt . . . . . . . . . . . . . . . . . 221

1. Der Turmdienst ...................... 223

2. Musikalische Dienste für die Bürger ......... 230

B. Öffentliche Konzerte .................. 233

V. Musik in Kirche und Schule . . . . . . . . . . . 241

A. Kirche und Orgel $\ldots \ldots \ldots \ldots \ldots \ldots \ldots \ldots \ldots \ldots \ldots \ldots \ldots$

B. Kirchenordnung und Kirchenmusik ........... 245

C. Das Amt des Organisten und Kantors ........... 251

D. Organisten, Präzeptoren, Provisoren, Kantoren . . . . 265

E. Kirchenmusik im 17. und 18. Jahrhundert . . . . . . . 289 
Mitglieder der Hofkapelle, Organisten und Kantoren in Kirchberg ............................... 301

Verwendete Archivalien ..................... 304

Literaturverzeichnis . . . . . . . . . . . . . . . . . . 309

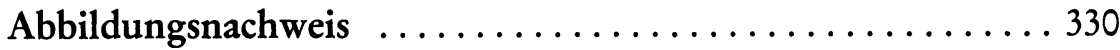

Personenregister $\ldots \ldots \ldots \ldots \ldots \ldots \ldots \ldots \ldots \ldots \ldots \ldots \ldots \ldots \ldots$ 


\section{Abkürzungen}

$\mathrm{AK}$

$B \ddot{u}$

DBA

$\mathrm{fl}$

HZN

LKA

Lebensbeschreibung

MGG
$=$ Archiv Kirchberg

= Büschel

= Deutsches biographisches Archiv: eine Kumulation aus 254 der wichtigsten biographischen Nachschlagewerke für den deutschen Bereich bis zum Ausgang des 19. Jabrbunderts, hrsg. von Bernhard Fabian. Microfiche Edition, München 1982.

$=$ Gulden

$=$ Hohenlohe-Zentralarchiv Neuenstein

= Landeskirchliches Archiv

$=$ Handschriftlicher Lebenslauf Carl Ludwig Junkers, aufbewahrt im Hohenlohe-Zentralarchiv Neuenstein.

= Die Musik in Geschichte und Gegenwart 1-17, hrsg. von Friedrich Blume, Kassel und Basel 1949-1986, Taschenbuchausgabe München und Kassel 1989.

Musikalische Lebensgeschichte = Musikalische Lebensgeschichte Karl Ludwig Junker's von ibm selbst beschrieben, in: Wirtembergisches Repertorium der Litteratur, 3. St., 1783, S. 442-462. 
Pfarrerbuch Württ.

Franken/Pfarreien

Pfarrerbuch Württ. Franken/ Kirchen- und Schuldiener

Privatrechnungen

Rechnungen

RGG
= Baden-Württembergisches Pfarrerbuch, Bd. II Württembergisch Franken, Teil 1 Die Pfarreien, bearb. von Max-Adolf Cramer, Stuttgart 1985.

= Baden-Württembergisches Pfarrerbuch, Bd. II Württembergisch Franken, Teil 2 Kirchen- und Schuldiener, bearb. von Otto Haug, Stuttgart 1981.

$=$ in Notizbücher eingetragene private Einnahmen und Ausgaben des Fürsten Christian Friedrich Carl zu Hohenlohe-Kirchberg, die eine unterschiedliche Anzahl von Jahren beinhalten.

$=$ mit Rechnungen werden die jährlich an Georgii (Ende April) beginnenden Rechnungsbücher der Hofhaltung, die unter unterschiedlichen Namen (Bsp. Hochgräflich Hohenloh-Kirchberg[ische] HausHofMeisterey GeldRechnungen, Hochfürstlich Hohenlohe-Kirchberg[ische] Hof-Cassa-Geldrechnungen) geführt und jährlich mit den Rechnungsbelegen in numerierte Bände zusammengefaßt wurden, einheitlich bezeichnet.

$=$ Die Religion in Geschichte und Gegenwart, hrsg. von Kurt Galling, 3. Aufl., Tübingen 1957-1965. 
RISM

Schreibalmanach

$\mathrm{X}$
$=$ Répertoire Internationale des Sources Musicales

$=$ Tagebücher Angehöriger des Hauses Hohenlohe, geführt in handelsüblichen Kalendern mit dem Titelblatt "Schreib-Almanach, Auf das Jahr Christi ...«. Die häufig gebrauchten Schreibalmanache des Fürsten Christian Friedrich Carl zu HohenloheKirchberg wurden nur mit der entsprechenden Datierung zitiert und auf die Bandnummern verzichtet.

$=$ Kreuzer

\section{Zur Quellenwiedergabe}

Die Texte werden mit Ausnahme der Anrede und Grußformel nicht zeilengetreu wiedergegeben. Die originale Schreibweise wird beibehalten. Das Schreiben der Doppelkonsonanten $\mathrm{mm}$ und $\mathrm{nn}$ als $\overline{\mathrm{m}}$ beziehungsweise $\bar{n}$ wird $\mathrm{zu}$ mm beziehungsweise $\mathrm{nn}$ vereinheitlicht. Ergänzungen werden durch eckige Klammern gekennzeichnet. Lediglich Verschleifungen der Wortendungen werden ohne Kennzeichnung aufgelöst. 